\title{
Alternating and direct current characterization and photoinduced absorption studies of modified conjugated polymer thin films
}

\author{
S. M. Lipson, J. N. Coleman, A. Drury, D. F. O'Brien, and W. J. Blau \\ Materials Ireland Polymer Research Centre, Department of Physics, Trinity College, Dublin 2, Ireland
}

A. J. Cadby

UCLA, Department of Chemistry and Biochemistry, 607 Charles E. Young Drive East, Box 951569,

Los Angeles, California 90095-1569

P. A. Lane

C. S. Draper Laboratory, 555 Technology Square, Cambridge, Massachusetts 02131

D. D. C. Bradley

Experimental Solid State Physics Group, Blackett Laboratory, Imperial College London, South Kensington Campus, London SW7 2BZ, United Kingdom

(Received 31 October 2003; accepted 8 March 2004)

\begin{abstract}
Control of interchain separation enables the optical properties of polymer thin films to be altered. We present a thin film preparation technique that increases the free volume fraction in spin coated polymer thin films resulting in reduced interchain interaction. The polaron yield, measured using photoinduced absorption, was significantly reduced as a direct result of the increased interchain separation, leading to a higher value for the measured photoluminescence efficiency. Impedance spectroscopy showed an increase in permittivity, probably due to greater polarizability. Increases approaching one order of magnitude were observed for direct current hole conductivity and mobility values. Space charge limited conduction analysis suggests a narrowing of the highest occupied molecular orbital band tail on treatment resulting in reduced trapping. Single layer light emitting devices prepared using this technique were found to be significantly brighter and to have longer operating lifetimes. (C) 2004 American Institute of Physics. [DOI: 10.1063/1.1719261]
\end{abstract}

\section{INTRODUCTION}

The availability of organic display ${ }^{1}$ technologies offers a viable alternative to existing display technologies in terms of cost, ease of fabrication, and viewing angle. The next logical step, an electrically pumped organic laser ${ }^{2,3}$ would revolutionize the world of optical communications and data storage. Some limitations however do exist in terms of low luminescence efficiency, low charge carrier mobility, and device stability. These and other issues have been highlighted as limiting factors in the fabrication of such a device. ${ }^{4-6}$

Thin polymer films exhibit significantly lower photoluminescence (PL) efficiencies than low concentration solutions of the same material. Aggregate formation at high concentration results in poor luminescence efficiency due to the formation of subgap states that can dissociate excitons. ${ }^{7,8}$ Previously, samples prepared by a thin film preparation technique, ${ }^{9}$ known as the treated technique, were calibrated for PL efficiency and photostability. ${ }^{10}$ The treatment was found to significantly improve these two properties by addressing the issue of aggregation. The treated films were spin coated in an inert atmosphere from degassed solutions and baked under vacuum. The film thickness increased by $\sim 30 \%$ and the PL efficiency by $27 \%$. These increases were calculated as a percentage of the values obtained for conventional polymer films that were prepared in ambient conditions from nondegassed solutions. Both of these were attributed to increased interchain separation brought about by the alteration of the film morphology due to the fabrication technique used. Such an increase would lead to a reduction in the formation of polarons and nonradiative quenching routes, resulting in increased PL efficiency. A thin film formation technique that addresses this polymeric property would have important implications for the future development of optical organic devices.

The main focus of this work is to study the electrical properties of the treated polymer films relative to conventional polymer films. Direct and alternating current measurement techniques were used to investigate any differences resulting from the changes in film morphology. The relationship between the polymer thin film preparation techniques and polaron formation was studied for both an untreated and a treated polymer film using photoinduced absorption spectroscopy. Light emitting devices (LEDs) were fabricated using both conventional and treated thin film preparation techniques to enable the effects of the increased PL efficiency and increased interchain separation to be studied in practical devices.

\section{MATERIALS AND EXPERIMENTAL METHODS}

The copolymer poly $(m-$ phenylenevinylene-co-2,5dioctyloxy-p-phenylenevinylene) (PmPV) was used in this work. The incorporation of the meta linkage on the phenylenevinylene co-unit is in an attempt to blue shift the absorption and luminescence spectra relative to $\operatorname{poly}(p-$ phenylenevinylene) (PPV) by restricting the conjugation 


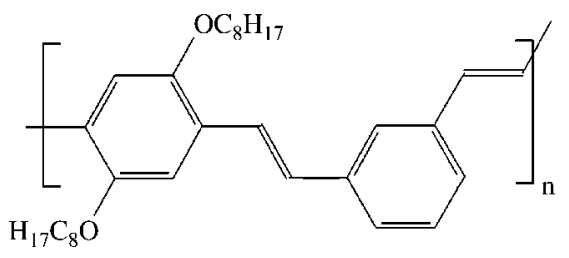

FIG. 1. The chemical structure of the repeat unit of the copolymer poly $(m-$ phenylenevinylene-co-2,5-dioctlyoxy-p-phenylenevinylene).

length and thus limiting exciton diffusion to lower energy segments of the polymer chain. A blue shift of the absorption and PL maxima compared to the corresponding all paraphenylenevinylene copolymer has been observed. ${ }^{11}$

The PmPV used in this work was synthesized by the Organic Materials Synthesis section of Materials Ireland Polymer Research Center in Trinity College Dublin. ${ }^{12}$ Two different synthesis reactions have been used with three solvents to give six different synthesis pathways for the polymer. ${ }^{13}$ The Horner-Emmons polycondensation reaction ${ }^{14}$ in dry toluene was used to synthesize the PmPV for this work. The chemical structure of PmPV is shown in Fig. 1.

Photoinduced absorption (PA) spectroscopy was performed to study the relationship between film forming techniques and polaron formation for neat thin films of PmPV prepared using the conventional and treated techniques. Both film types were prepared from $25 \mathrm{~g} / \mathrm{l}$ toluene solutions of PmPV and spin coated at $400 \mathrm{rpm}$ on Spectrosil B substrates. The samples were excited by an amplitude-modulated pump beam and changes in the transmission of a probe beam were measured by lock-in amplification of a photodetector. The $457.9 \mathrm{~nm}$ line of an argon ion laser at a power density of 30 $\mathrm{mW} \mathrm{cm}{ }^{-2}$ was used as the pump beam and a monochromated tungsten white light source was used as the probe. The probe light transmitted through the sample was passed through a monochromator set to the same wavelength as the probe beam. The use of multiple detectors and gratings enabled the PA spectrum to be investigated between $500-1800 \mathrm{~nm}$. The pump beam was modulated at $120 \mathrm{~Hz}$ by an acousto-optical modulator and the spectra were measured in a cryostat, under vacuum at $80 \mathrm{~K}$. The PA spectrum is defined as the normalized change in the transmission of the probe beam $(\Delta T / T)$. The lock-in amplifier simultaneously measures the in-phase and quadrature signals, which can be compared to determine the excitation lifetime ${ }^{15}$ and correlate different PA bands. By measuring the dependence of the PA signal on the laser power it is possible to identify the species responsible for the observed absorption.

For electrical characterization, patterned bottom contacts were deposited on glass substrates by sputtering. PmPV films were then spin coated on top of the bottom contact from a 25 $\mathrm{g} / \mathrm{l}$ toluene solution, at $400 \mathrm{rpm}$ for $60 \mathrm{~s}$. Finally the top contacts were deposited, by thermal evaporation or sputtering, depending on the metal being deposited. Electron and hole only currents were obtained using aluminum $(\mathrm{Al})$ and platinum $(\mathrm{Pt})$ contacts, respectively. Their work functions are approximately $4.28 \mathrm{eV}$ and $5.65 \mathrm{eV}$, respectively, varying slightly depending on deposition parameters. LEDs were prepared using indium tin oxide (ITO) and aluminum as the

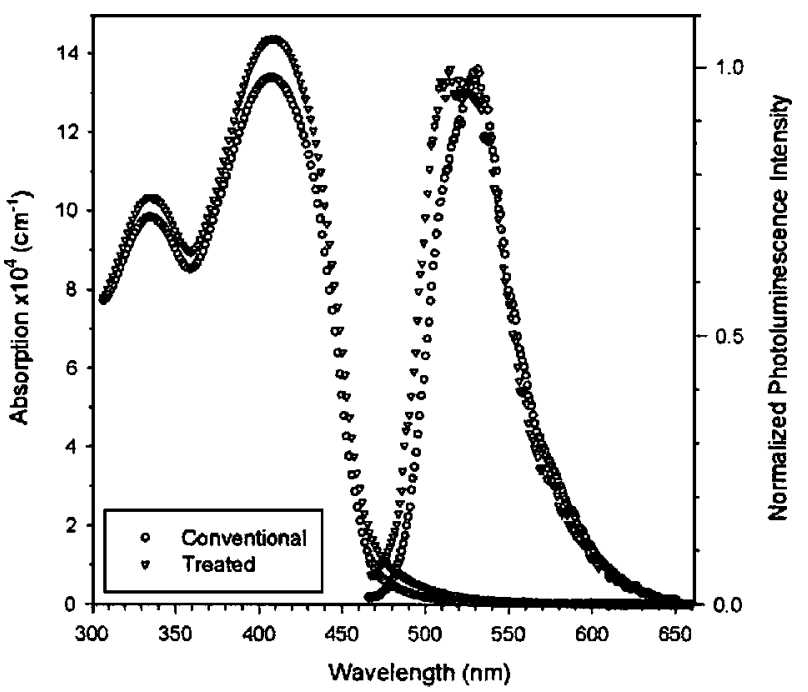

FIG. 2. Absorption and normalized photoluminescence of PmPV in the solid state for conventional (open circle) and treated (open inverted triangle) films.

hole and the electron injecting electrodes, respectively. All devices were tested in a purpose built test rig. Contacts were made using conductive silver paint and pressure probes. The probes were connected to a Keithley 2400 source measure unit for direct current (dc) measurements and a Zahner Electric IM6e Impedance Spectrum Analyzer for alternating current (ac) measurements. The devices were tested in an argon atmosphere. For ac measurements a $5 \mathrm{mV}$ alternating voltage was applied in all measurements. The initial run was recorded at $0 \mathrm{~V}$ dc bias, while subsequent runs were recorded with either positive or negative dc bias incrementing in $1 \mathrm{~V}$ steps to $\pm 4 \mathrm{~V}$. Data was recorded between $0.1 \mathrm{~Hz}$ and 1 $\mathrm{MHz}$ for all measurements. Devices were reverse biased, while measuring the dc biased $I-V$ characteristics, to check for symmetrical $I-V$ behavior, which is to be expected from unipolar devices.

\section{OPTICAL CHARACTERIZATION}

The ultraviolet-visible absorption spectra of the conventional and treated films are shown in Fig. 2. It is immediately evident that the absorption coefficient $(\alpha)$ is larger in the treated film. With a $\sim 30 \%$ film thickness increase in the treated films resulting from an increased interchain separation, a reduction in $\alpha$ would be expected. However the observed behavior may be due to an increase in the oscillator strength caused by the reduction of interchain interactions associated with increased free volume in the treated film. Changes in the orientation of polymer chains within the plain of the film could influence the absorption as measured using normal incidence light and may also be a contributory factor in the observed differences. The normalized photoluminescence spectra are also shown in Fig. 2, with a $7 \mathrm{~nm}$ broadening towards the blue evident in the treated film. It is noted that this results from the quenching of the $510 \mathrm{~nm}$ peak in the conventional film relative to the treated film. Morphology changes in the treated film would account for this. An in- 


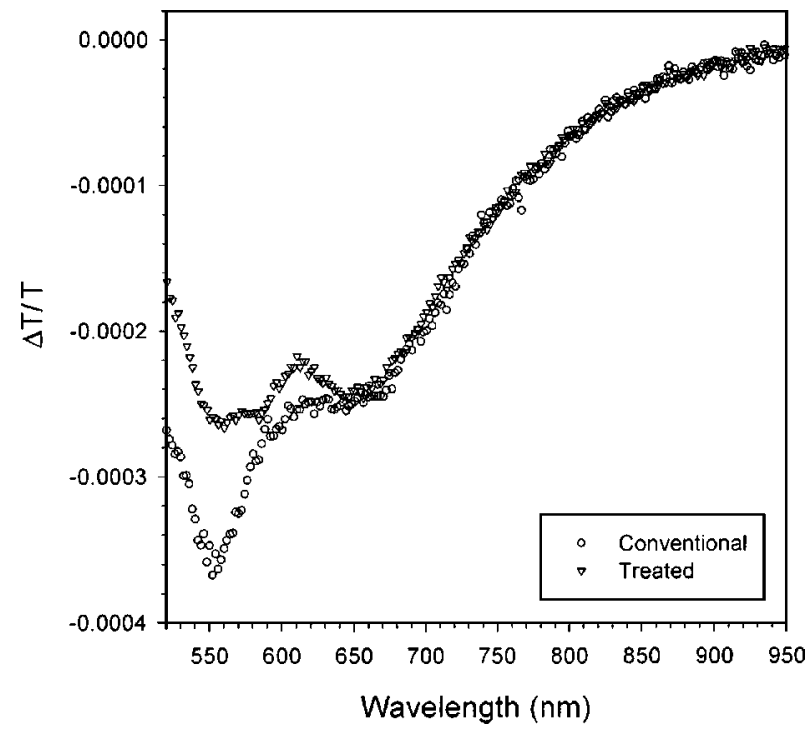

FIG. 3. Quadrature photoinduced absorption spectra for the conventional (open circle) and the treated (open inverted triangle) films. The reduction in the polaron peak at $550 \mathrm{~nm}$ in the treated film is clearly evident relative to the conventional film.

crease in interchain separation would result in the formation of fewer aggregates, resulting in a broadening towards shorter wavelengths.

The strongest evidence supporting the argument for increased interchain separation in the treated films comes from the photoinduced absorption experiment. Figure 3 clearly shows a significant reduction in the polaron population of the treated film relative to the conventional film. This is evident in the change in intensity of the peak at $550 \mathrm{~nm}$. The two absorptions shown in Fig. 3 at $550 \mathrm{~nm}$ and $660 \mathrm{~nm}$ were assigned to polarons and triplet excitons, respectively, by measuring their dependence on the pump beam power. Lifetimes were measured to be $0.44 \pm 0.02 \mathrm{~ms}$ for both excitations in both film types at $80 \mathrm{~K}$. It should be pointed out that quadrature plots are shown in Fig. 3, as correcting the inphase plots for PL proved inaccurate. No other PA peaks were observed beyond $950 \mathrm{~nm}$. Polarons in PmPV are predominantly formed through interchain separation as previously reported for neat PmPV films and PmPV/polystyrene blends. ${ }^{10,16}$ This result indicates that there is increased interchain separation in the treated film, agreeing with film thickness measurements for treated films relative to conventional films. The reduction in polaron formation contributes towards the increased PL efficiency measured in the treated film relative to the conventional film. The PL efficiency was measured with excitation at $354 \mathrm{~nm}$ using an integrating sphere.

The pump wavelength used for the PA was in the tail of the absorption for PmPV. This could give rise to differences in the PL compared to spectra taken with shorter pump wavelengths, due to the excitation of aggregate species, thus influencing the PA absorptions measured. It was however noted that PL spectra for PmPV taken at the absorption maximum of $407 \mathrm{~nm}$ had the same line shape as spectra taken at 457.9 $\mathrm{nm}$. It should also be considered that the increases in free volume associated with the treated film would result in a lower refractive index. This would increase the light output coupling efficiency by increasing the critical angle for waveguiding and could also be a contributory factor in the measured PL efficiency increase.

These results correlate reduced polaron photogeneration with enhanced PL quantum yield and increased free volume within the polymer film. Interchain aggregation can enhance polaron photogeneration, ${ }^{17}$ which in turn will reduce the PL quantum yield. Polarons quench singlet excitons ${ }^{18}$ and if the excited state absorption and PL spectra overlap, excited state absorption will also reduce the PL yield. The strong overlap of the excited state absorption due to polarons and the fluorescence in PmPV makes solid state lasing applications unlikely for this material. This is highly evident in amplified spontaneous emission (ASE) experiments. Spectral narrowing under high intensity laser illumination in ASE waveguiding geometries has not been observed in PmPV despite having been observed in other PPV derivatives. ${ }^{19}$ This has been attributed to the quenching of fluorescence due to polaron absorption and high waveguide losses due to scattering caused by the polycrystalline nature of PmPV. ${ }^{20,21}$ Lasing has however been reported for PmPV in solution, ${ }^{22}$ supporting the argument that interchain interactions reduce with increasing interchain separation.

\section{AC AND DC ELECTRICAL CHARACTERIZATION}

The focus of this section is to investigate the effect of treatment on the electrical properties of the polymer films. To this end dc current voltage measurements and ac impedance studies were carried out at room temperature. The frequency dependent electrical behavior of a semiconductor is described by the complex impedance $Z(\omega)$ :

$$
Z(\omega)=R(\omega)+\iota X_{\mathrm{c}}=R(\omega)+\iota \frac{1}{\omega C(\omega)},
$$

where $R(\omega)$ is the resistance, $X_{\mathrm{c}}(\omega)$ is the capacitive reactance, and $C(\omega)$ is the capacitance. Alternatively, a semiconductor film sandwiched between electrodes in a planar geometry can be modeled as an equivalent circuit consisting of a resistor and capacitor in parallel with a shunt resistor $\left(R_{\mathrm{S}}\right)$ in series. The total complex impedance of the equivalent circuit is then given by

$$
Z=R_{\mathrm{s}}+\frac{R-\iota \omega C R^{2}}{1+(\omega C R)^{2}} .
$$

The raw frequency dependent real and imaginary impedance data was fitted to Eq. (2) using a Levenberg-Marquardt nonlinear least squares fitting routine ${ }^{23,24}$ with $R$ and $C$ as free parameters. $\operatorname{Re} Z$ and $\operatorname{Im} Z$ were fitted individually and simultaneously, resulting in three solutions for $R$ and $C$. An example of a $\operatorname{Re} Z$ and $\operatorname{Im} Z$ vs angular frequency plot and the least squares fit, for platinum electrodes, is shown in Figs. 4(a) and 4(b) for a conventional and treated film, respectively. In both graphs the dc bias is $0 \mathrm{~V}$. These plots have been normalized for film thickness and clearly show that the resistance of the treated film is lower than that of the conventional film. This can be gauged by the value of $\operatorname{Re} Z$ at low frequency. It is noted that the individual fits are obscured 


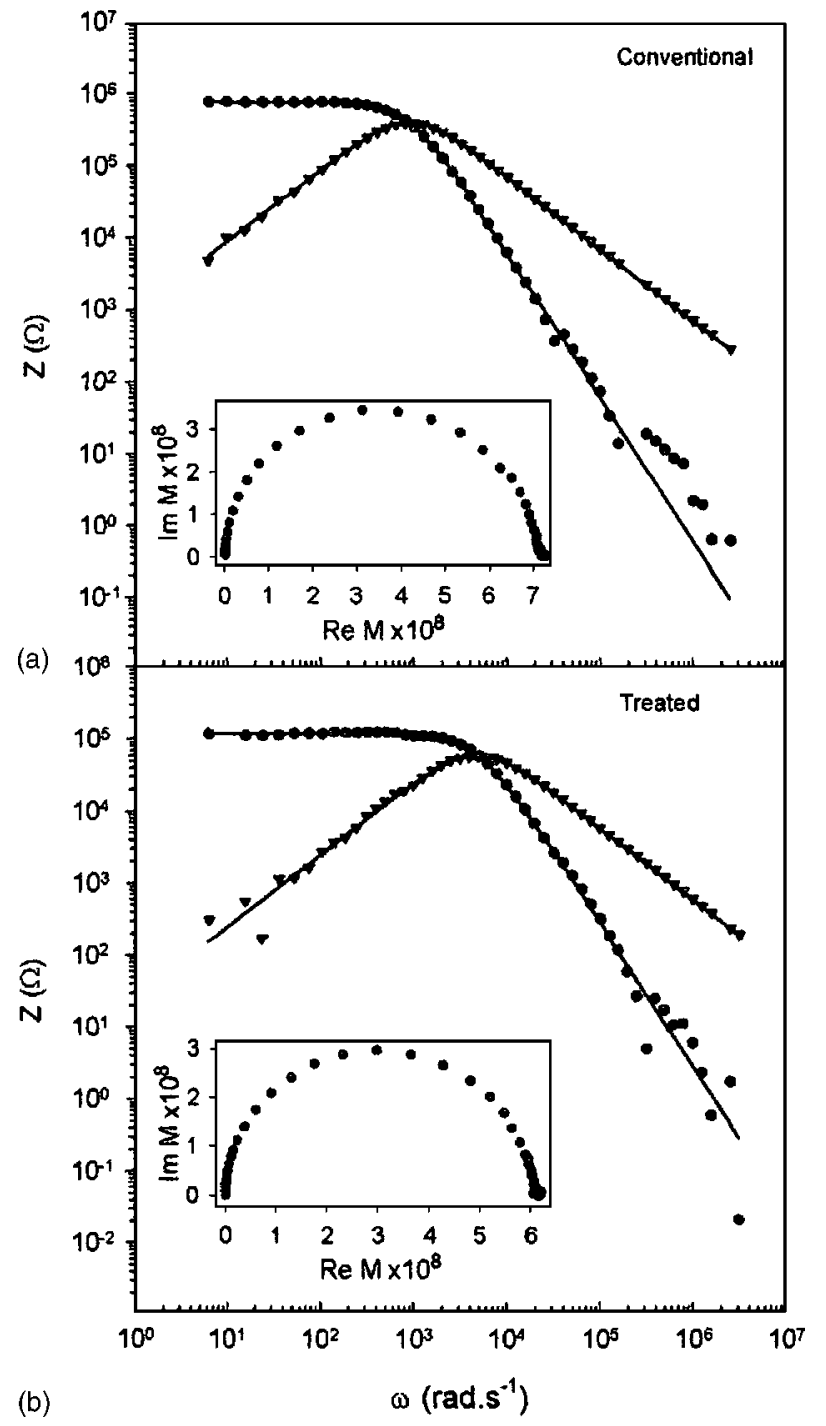

FIG. 4. $\operatorname{Re} Z$ (solid circles) and $|\operatorname{Im} Z|$ (solid inverted triangles) vs angular frequency, for (a) a conventional and (b) a treated PmPV film. The individual (dotted line) and simultaneous (solid line) fits are also shown. Both devices were prepared using platinum electrodes. The ac bias is $5 \mathrm{mV}$ and the dc bias is $0 \mathrm{~V}$. Both plots have been normalized for film thickness. It is noted that the individual fits are obscured in the plot due to the excellent correlation between the simultaneous fits and the individual fits to $\operatorname{Re} Z$ and $\operatorname{Im} Z$. The Cole-Cole plot for the data is shown in the inset.

in the plot due to the excellent correlation between the simultaneous fits and the individual fits to $\operatorname{Re} Z$ and $\operatorname{Im} Z$.

If a significant energy mismatch exists between the Fermi energy of an electron (hole) injecting electrode and the lowest unoccupied molecular orbital (LUMO) [highest occupied molecular orbital (HOMO)], a Schottky barrier may be formed at the interface. Depending on the relative positions of the Fermi energy and the LUMO (HOMO) the subsequent alignment of the Fermi energies may result in the formation of a depletion region due to the presence of impurity dopants. As the work function of $\mathrm{Al}$ is greater than the electron affinity for organics in general, depletion region formation is more likely than charge accumulation. When the data shown in Fig. 4 is presented in Cole-Cole format (inset), a single semicircle is observed. This suggests that the film acts as a single layer, suggesting either the absence of a depletion re-

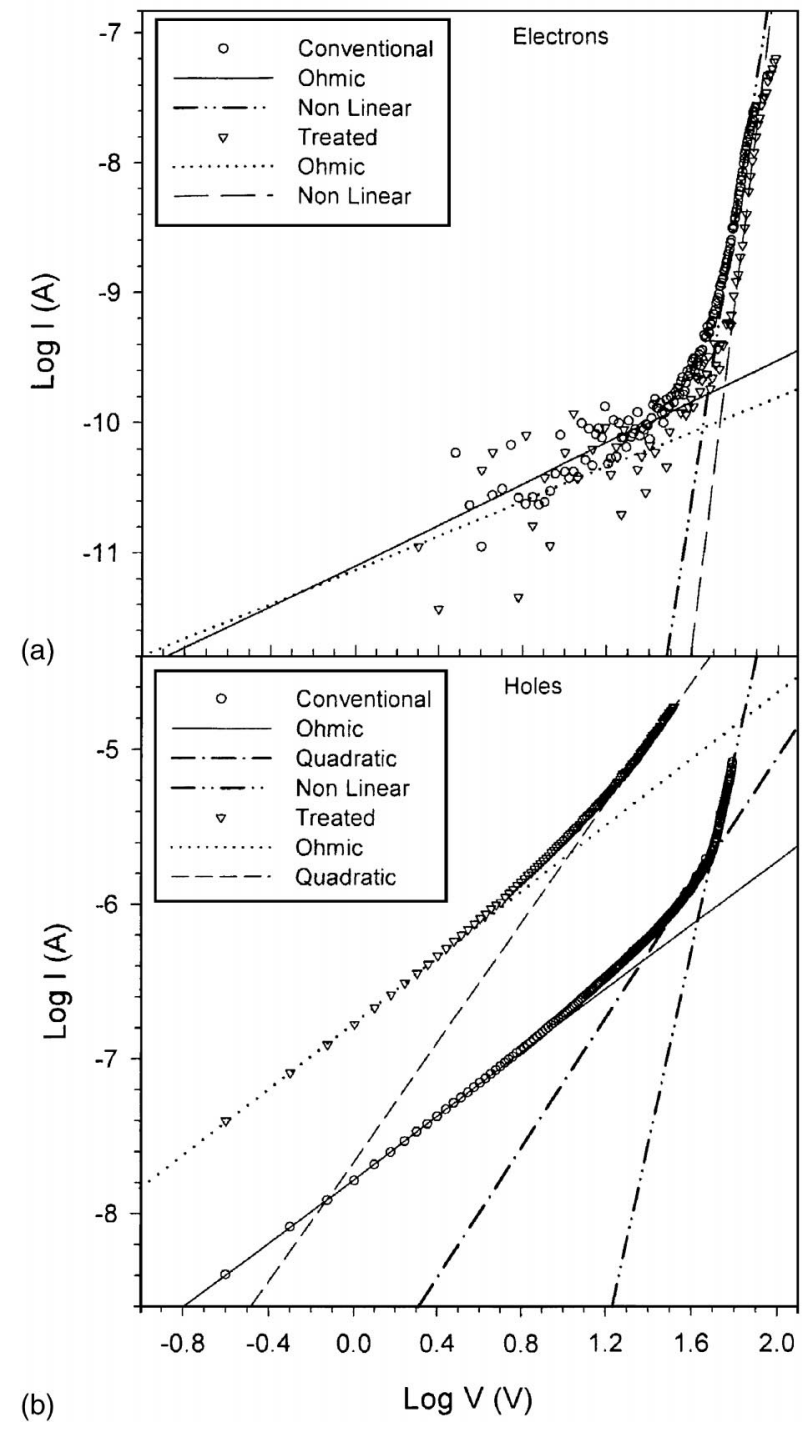

FIG. 5. (a) $\log I$ vs $\log V$ plot for a conventional (open circle) and a treated (open inverted triangle) PmPV film using aluminum electrodes. (b) $\log I$ vs $\log V$ plot for a conventional (open circle) and a treated (open inverted triangle) PmPV film using platinum electrodes. Linear, quadratic, and nonlinear fits to the data are shown.

gion or that the film is fully depleted. In order to rule out the latter possibility the capacitance was measured as a function of applied voltage. Application of a voltage to such polymermetal contacts acts to change the width of any depletion region present, thus altering the capacitance. However, in the voltage range studied the capacitance was found to be independent of voltage for both device types. This is strong evidence against the presence of a Schottky barrier in any of the samples studied here suggesting reasonably Ohmic contacts in all cases. This is supported by current-voltage $(I-V)$ data presented in Fig. 5.

The real and imaginary impedance data shown in Fig. 4 can be transformed to give the frequency dependent capacitance. For all films the capacitance was constant in the frequency range $12 \mathrm{~Hz}-1 \mathrm{MHz}$. Variations were observed below $12 \mathrm{~Hz}$. This allows the calculation of the relative permittivity $\left(\varepsilon_{\mathrm{r}}\right)$ of the two film types both from the frequency dependent capacitance and from the fits of the im- 
pedance to Eq. (2) with both methods giving very good agreement. The values calculated show a clear distinction between the conventional and the treated film types. The values measured were $6.2 \pm 0.4$ and $7.5 \pm 0.6$, respectively. This represents a $21 \%$ increase in the value of $\varepsilon_{\mathrm{r}}$ in the treated film. Changes in the macroscopic parameter $\varepsilon_{\mathrm{r}}$ directly correlate to changes in the microscopic polarizability of a material as described by the Clausius-Mossotti equation. The higher value for $\varepsilon_{\mathrm{r}}$ measured in the treated films is evidence of increased polarizability. This is almost certainly due to a reduction of interchain interactions as a result of increased interchain separation. ${ }^{25}$ Thus the increase in $\varepsilon_{\mathrm{r}}$ confirms the increased interchain separation inferred from the film thickness, absorption, PL, and photoinduced absorption measurements.

It should be noted that the values measured for $\varepsilon_{\mathrm{r}}$ are larger than values previously reported ${ }^{26,27}$ for conjugated polymers. It is however noted that some authors have also reported similar values ${ }^{28,29}$ to the ones presented in this work. A possible contribution from a fluctuating permanent dipole associated with the presence of alkoxy side chains may account for the larger values, although a more detailed study is needed to establish the exact nature of any possible sidechain contribution.

The dc current-voltage graphs for conventional and treated unipolar devices are presented in Figs. 5(a) and 5(b) for electron only and hole only devices, respectively. The graphs are plotted on a logarithmic scale. For the electron only samples (Al/PmPV/Al), the $I-V$ curves for both conventional and treated PmPV are almost identical, with any differences due to film thickness differences. In both cases an approximately Ohmic $(I \propto V)$ region is observed below $\sim 40$ $\mathrm{V}$. This is followed by a highly nonlinear region that follows a power law $I \propto V^{\mathrm{m}+1}, m+1 \approx 11$. This result is characteristic of the presence of an exponential trap distribution extending into the band gap from the LUMO band edge. The characteristic depth of this distribution is given by mkT, which is calculated to be $0.25 \mathrm{eV}$. The similarity between the conventional and treated data sets suggests that the treatment technique has very little effect on the electron transport. However it should be pointed out that electron transport is not degraded by the film morphology changes. In the case of the hole only $(\mathrm{Pt} / \mathrm{PmPV} / \mathrm{Pt})$ data however significant differences are observed between conventional and treated samples. In both cases, at low voltage an Ohmic region is observed. However for the treated sample, the conductivity is $\sigma_{\mathrm{T}}$ $=3.6 \times 10^{-8} \mathrm{~S} / \mathrm{m}$ which is a factor of 5 larger than the conductivity of the conventional film, $\sigma_{\mathrm{C}}=6.6 \times 10^{-9} \mathrm{~S} / \mathrm{m}$. When the voltage exceeds approximately $\sim 20 \mathrm{~V}$ the $I-V$ curve becomes nonlinear with a slope on a log-log plot of approximately 2. This quadratic dependence of current on voltage is consistent with unipolar space charge limited conduction first described by Mott and Gurney in 1940: ${ }^{30}$

$$
J=9 \varepsilon \mu_{\mathrm{eff}} V^{2} / 8 d^{3}
$$

where $J$ is the current density, $\varepsilon$ is the permittivity of the polymer, $\mu_{\text {eff }}$ is the effective mobility, $V$ is the applied voltage, and $d$ is the thickness of the polymer film. This allows the calculation of effective mobility for both conventional and treated films. For the treated film the mobility was 1.3 $\times 10^{-11} \mathrm{~m}^{2} / \mathrm{V} \mathrm{s}$ which is a factor of 6 larger that the conventional value of $2.1 \times 10^{-12} \mathrm{~m}^{2} / \mathrm{V} \mathrm{s}$. The final difference between samples is that the conventional sample displays a sharp increase in current at approximately $40 \mathrm{~V}$. This increase is linear on a log-log plot with slope $m+1=6$. As in the electron only case this is consistent with the presence of an exponential trap distribution extending into the band gap from the HOMO with a characteristic trap depth of $0.13 \mathrm{eV}$. No evidence of this distribution is observed for the treated sample. A deeper understanding of this dc analysis of trap distributions can be attained through film thickness and temperature dependent measurements.

As discussed previously analysis of the optical and ac electrical data suggests the major effect of the treatment technique is to modify the polymer morphology so as to lead to the introduction of more free volume. This in turn reduces polymer interchain interactions. It is likely that for the conventional film, these interactions act to modify the polymer density of states such that the HOMO band edge is broadened into a band tail with approximately an exponential distribution. It is possible that the reduction in interchain interactions in the treated film results in less broadening of the bandtail. As a result a high exponent power law region is not seen in the hole $I-V$ curves for the treated sample. In addition the reduction in traps in the treated sample would explain the increase in effective mobility in that material. The effective mobility is given by the product of the intrinsic mobility $\left(\mu_{0}\right)$ and the fraction of free charge:

$$
\mu_{\mathrm{eff}}=\mu_{0}\left[N_{\mathrm{F}} /\left(N_{\mathrm{F}}+N_{\mathrm{T}}\right)\right],
$$

where $N_{\mathrm{F}}$ is the number density of free carriers and $N_{\mathrm{T}}$ is the number density of trapped carriers. This suggests that the increase in mobility between conventional and treated samples is due to the reduction in the number density of trapped charge in the latter.

However it should be pointed out that the treatment technique alters the density of states at the HOMO band edge in the case of hole only transport but no evidence of any alteration is seen for the LUMO band edge in the electron only samples.

LEDs were prepared using both the conventional and the treated film preparation techniques to investigate if the improvement in photoluminescence efficiency and the increased hole mobility in treated films translates into brighter and more efficient LEDs. The plots of $\log I$ vs $\log V$ and output light intensity vs $\log V$ are shown in Fig. 6. The current voltage curves both show a low slope region followed by a high slope region with a transition voltage of 28.2 and $22.4 \mathrm{~V}$ for the conventional and treated devices, respectively. The high slope regions have approximately the same exponent $(m+1 \approx 12)$ and indicate the presence of an exponential trap distribution, which is typical of such organic devices and has been widely reported. ${ }^{31}$ The presence of such a high slope region in the treated sample is probably at least in part due to the exponential electron trap distribution. It can be seen that the turn on voltage for light emission is slightly lower in the treated device at $34 \mathrm{~V}$ compared to $36 \mathrm{~V}$ in the conventional device. At the device failure voltage of $50 \mathrm{~V}$ the 


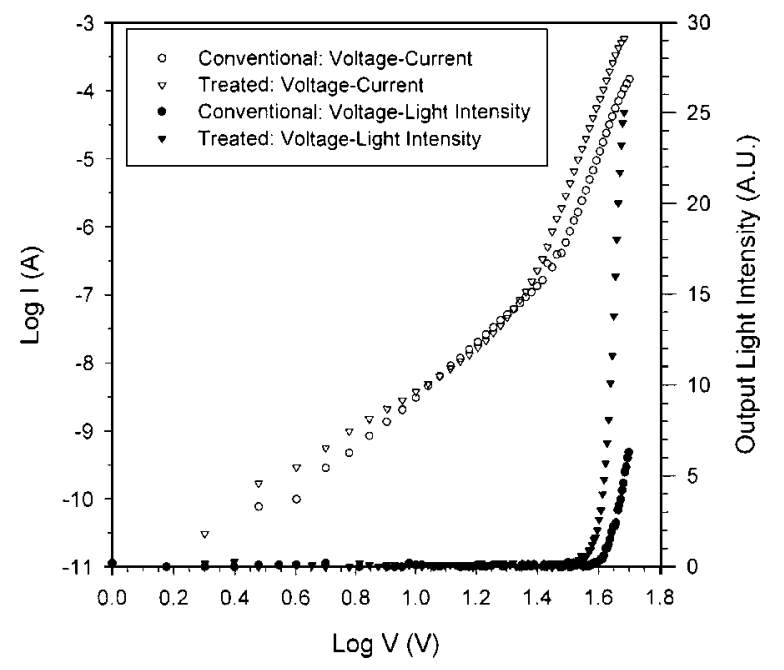

FIG. 6. $\log I$ vs $\log V$ for conventional (open circles) and treated (open inverted triangles) PmPV LEDs. Also shown is the output light intensity vs $\log V$ for the conventional (solid circles) and treated (solid inverted triangles) PmPV LEDs.

intensity of the light emission from the treated device is over four times greater than that in the conventional device. A plot of output light intensity vs current density $(J)$ is shown in Fig. 7 for the two device types and enables the relative efficiencies to be established, in terms of light output per unit current. At low current densities no discernible differences are evident. With the increased PL efficiency in the treated devices an increase in efficiency would be expected. At current densities above $40 \mathrm{Am}^{-2}$ the efficiency of the conventional device starts to drop off relative to the treated device. This saturation does not occur in the treated film until a current density of $190 \mathrm{Am}^{-2}$ is achieved. This shows that at the top end of the operational capacity of conventional devices, the treated devices are starting to become more efficient. The improved hole mobility resulting from a reduction in trapping sites in the treated films is thought to contribute

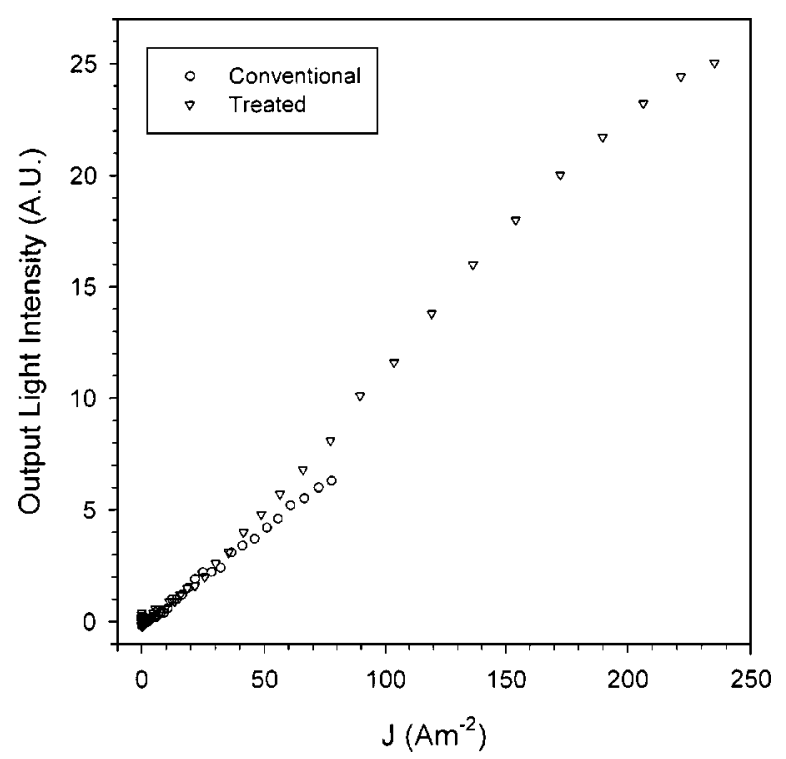

FIG. 7. Relative current efficiencies for conventional (open circles) and treated (open inverted triangles) PmPV LEDs. to these results. It was observed that the treated devices were also significantly more stable and had longer operating lifetimes.

\section{CONCLUSIONS}

Photoinduced absorption measurements show a clear reduction in the formation of polarons in the treated films of PmPV. This result, coupled with the film thickness, absorption, photoluminescence spectral and efficiency measurements, supports the argument presented for an increased interchain separation in the treated films due to changes in the film morphology emanating from the preparation of the films. This suggests that by controlling thin film processing the photophysical properties of thin films can be engineered. In this work we have shown that increasing interchain separation can significantly reduce the number of polarons formed in thin films of PmPV. This decrease in polaron formation results in a measured photoluminescence efficiency increase of $27 \%$. This work has important implications for understanding further the photophysics of luminescent polymers and more immediately in developing a successful polymer for use as an emissive layer in an electrically pumped organic laser.

Electrical measurements on PmPV thin films have enabled the effect of increased interchain separation on the electrical properties to be characterized. The important point is that the treatment of PmPV films is not detrimental to the performance of electrical devices. The calculation of the relative permittivity enables the polarizability of polymeric materials to be compared. The value measured for the treated film was $21 \%$ larger than that in the conventional film, indicating a larger polarizability. This is an important result as it supports the findings of the PA experiment through an independent experimental technique. The current-voltage characteristics showed the presence of an exponential hole trap distribution in the conventional but not the treated samples. An increase of nearly an order of magnitude in the hole conductivity and mobility in treated films of PmPV was also observed, again indicating the reduction of trapping. The dc electrical properties for electron only currents were the same for both film types. The fabrication of single layer LEDs using both film preparation techniques showed that for current densities above $40 \mathrm{Am}^{-2}$ a significant improvement in output light intensity efficiency was achieved. In conclusion the preparation of treated devices from PmPV had a positive effect on device performance. This is shown by the higher hole conductivities that were measured and the higher value of the output light intensity and saturation current density measured in the treated LEDs.

\section{ACKNOWLEDGMENTS}

The authors wish to acknowledge the financial support of the Irish Higher Education Authority and the COST 518 project.

${ }^{1}$ H. Kobayashi, S. Kanbe, S. Seki, H. Kigchi, M. Kimura, I. Yudasaka, S. Miyashita, T. Shimoda, C. R. Towns, J. H. Burroughes, and R. H. Friend, Synth. Met. 111-112, 125 (2000).

${ }^{2}$ Commission of the European Community ESPRIT project 28580 (LUPO). 
${ }^{3}$ F. Hide, M. A. Diaz-Garcia, B. J. Schwartz, M. R. Andersson, Q. B. Pei, and A. J. Heeger, Science 273, 1833 (1996).

${ }^{4}$ N. Tessler, Adv. Mater. (Weinheim, Ger.) 11, 363 (1999).

${ }^{5}$ G. Kranzelbinder and G. Lising, Rep. Prog. Phys. 63, 729 (2000).

${ }^{6}$ N. Tessler, N. T. Harrison, D. S. Thomas, and R. H. Friend, Appl. Phys. Lett. 73, 732 (1998)

${ }^{7}$ L. J. Rothberg, M. Yan, F. Papadimitrakopoulos, M. E. Galvin, E. W. Kwock, and T. M. Miller, Synth. Met. 80, 41 (1996).

${ }^{8}$ B. J. Schwartz, F. Hide, M. R. Andersson, and A. J. Heeger, Chem. Phys. Lett. 265, 327 (1997).

${ }^{9}$ S. M. Lipson, D. F. O’Brien, H. J. Byrne, A. P. Davey, and W. J. Blau, Thin Solid Films 370, 262 (2000).

${ }^{10}$ S. M. Lipson, A. J. Cadby, P. A. Lane, D. F. O’Brien, D. D. C. Bradley, and W. J. Blau, Chem. Month. 132, 151 (2001).

${ }^{11}$ D. F. O'Brien, A. Bleyer, D. G. Lidzey, D. D. C. Bradley, T. Tsutsui, J. Appl. Phys. 82, 2662 (1997).

${ }^{12}$ A. P. Davey, A. Drury, S. Maier, H. J. Byrne, and W. J. Blau, Synth. Met. 103, 2478 (1999).

${ }^{13}$ A. Drury, S. Maier, M. Ruther, and W. J. Blau, J. Mater. Chem. 13, 485 (2003).

${ }^{14}$ H. Rost, A. Teuschel, S. Pfeiffer, and H. H. Horhold, Synth. Met. 84, 269 (1997).

${ }^{15}$ C. Botta, S. Luzzati, R. Tubino, D. D. C. Bradley, and R. H. Friend, Phys. Rev. B 48, 14809 (1993).

${ }^{16}$ P. A. Lane, A. J. Cadby, H. Mellor, S. J. Martin, D. G. Lidzey, D. D. C. Bradley, S. M. Lipson, D. F. O’Brien, and W. J. Blau, Phys. Rev. B 62, 15718 (2000).

${ }^{17}$ T. Q. Nguyen, I. B. Martini, J. Liu, and B. J. Schwartz, J. Phys. Chem. B 104, 237 (2000).
${ }^{18}$ P. A. Lane, X. Wei, and Z. V. Vardeny, Phys. Rev. Lett. 77, 1544 (1996).

${ }^{19}$ M. D. McGehee, R. Gupta, S. Veenstra, E. K. Miller, M. A. Diaz-Garcia, and A. J. Heeger, Phys. Rev. B 58, 7035 (1998).

${ }^{20}$ R. Resel, B. Tertinek, S. Tasch, A. Davey, W. Blau, H. H. Horhold, R. Rost, and G. Leising, Synth. Met. 101, 96 (1999).

${ }^{21}$ B. McCarthy, R. Czerw, A. Strevens, A. P. Davey, D. L. Caroll, and W. J. Blau, Mater. Res. Soc. Symp. Proc. 598, BB11.34.1-6 (2000).

${ }^{22}$ W. Holzer, A. Penzkofer, S.-H. Gong, A. Bleyer, and D. D. C. Bradley, Adv. Mater. (Weinheim, Ger.) 8, 974 (1996).

${ }^{23}$ D. W. Marquardt, J. Soc. Ind. Appl. Math. 11, 431 (1963).

${ }^{24}$ Numerical Recipes in C: The Art of Scientific Computing, 2nd ed. (Cambridge University Press, Cambridge, 1992).

${ }^{25}$ T.-Q. Nguyen, R. C. Kwong, M. E. Thompson, and B. J. Schwartz, Appl. Phys. Lett. 76, 2454 (2000).

${ }^{26}$ J. Plocharski, H. Drabik, H. Wycislik, and T. Ciach, Synth. Met. 88, 139 (1997).

${ }^{27}$ A. J. Campbell, D. D. C. Bradley, and H. Antoniadis, J. Appl. Phys. 89, 3343 (2001).

${ }^{28}$ C. Y. Lee, H. M. Kim, J. K. Park, Y. S. Gal, J. I. Jin, and J. Joo, Synth. Met. 117, 109 (2001).

${ }^{29}$ R. D. Schaller, L. F. Lee, J. C. Johnson, L. H. Haber, R. J. Saykally, J. Vieceli, I. Benjamin, T.-Q. Nguyen, and B. J. Schwartz, J. Phys. Chem. B 106, 9496 (2002).

${ }^{30}$ N. F. Mott and R. W. Gurney, Electronic Processes in Ionic Crystals (Dover, New York, 1940)

${ }^{31}$ A. J. Campbell, M. S. Weaver, B. D. G. Lidzey, and D. D. C. Bradley, J. Appl. Phys. 84, 6737 (1998). 